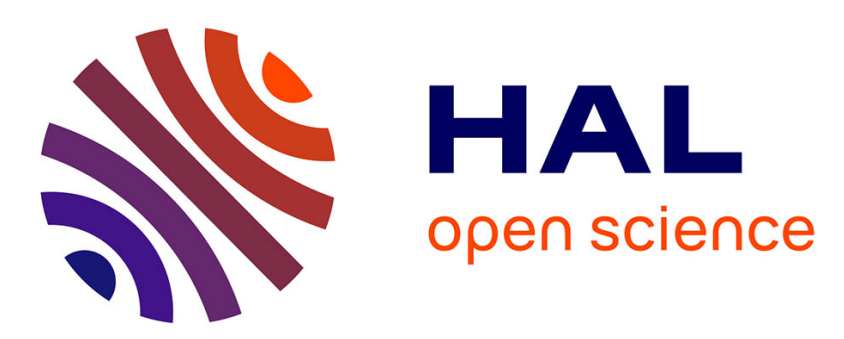

\title{
The Hidden Robot Concept: a Tool for Control Analysis and Robot Control-based Design
}

Sébastien Briot, Victor Rosenzveig, Philippe Martinet

\section{To cite this version:}

Sébastien Briot, Victor Rosenzveig, Philippe Martinet. The Hidden Robot Concept: a Tool for Control Analysis and Robot Control-based Design. Advances in Robot Kinematics, Jun 2014, Ljubljana, Slovenia. hal-00955105

\section{HAL Id: hal-00955105 https://hal.science/hal-00955105}

Submitted on 25 Jun 2019

HAL is a multi-disciplinary open access archive for the deposit and dissemination of scientific research documents, whether they are published or not. The documents may come from teaching and research institutions in France or abroad, or from public or private research centers.
L'archive ouverte pluridisciplinaire $\mathbf{H A L}$, est destinée au dépôt et à la diffusion de documents scientifiques de niveau recherche, publiés ou non, émanant des établissements d'enseignement et de recherche français ou étrangers, des laboratoires publics ou privés. 


\title{
The Hidden Robot Concept: a Tool for Control Analysis and Robot Control-based Design
}

\author{
Sébastien Briot, Victor Rosenzveig, and Philippe Martinet
}

\begin{abstract}
Exteroceptive sensors can be used to estimate the robot pose in order to suppress inaccuracies coming from the accumulation of modelling errors when using the classical control approach. In some cases, it is impossible to directly observe the end-effector. Thus we can replace it efficiently by the observation of the legs directions. However, with such an approach, unusual results were recorded, namely: (i) the possibility of controlling the robot by observing a limited number of legs, and (ii) in some cases, the robot does not converge to the desired end-effector pose, even if the observed leg did. These results can be explained through the use of the hidden robot concept, which is a tangible visualisation of the mapping between the observed leg direction space and Cartesian space. In the present paper, it is explained (1) why the tools used in mechanical design can be efficently applied in control analysis via the use of the hidden robot concept which is, in our opinion, a way to unify the analysis of the mechanical and control performances and (2) why we believe that the hidden robot concept must be used to modify the robot design methodologies in order to include control-based performance indices.
\end{abstract}

Key words: Parallel robots, Control analysis, Robot design.

\section{Introduction}

Sensor-based control approaches have proven to be more efficient than model-based control approaches when accuracy is required in robotized industrial applications [9]. The most usual approach is to observe the robot end-effector pose through the use of a camera. However, for some operations, such as the milling of materials,

This work was supported by the French ANR project ARROW (ANR 2011BS3 006 01). Sébastien Briot, Victor Rosenzveig and Philippe Martinet IRCCyN, UMR CNRS 6597, France, and École Centrale de Nantes, France

e-mail: \{Sebastien.Briot,Victor.Rosenzveig,Philippe.Martinet\}@irccyn.ec-nantes.fr 
it is not possible to observe the end-effector. An innovative vision-based control approach that can be used on parallel robots has been proposed in [2]. It is based on the fact that parallel robot links are usually made of rectilinar cylindrical rods that can be easily detected in the camera space. The information being acquired through an external sensor, this technique allows to estimate indirectly the pose of the end-effector from it.

Servoing through the robot leg observation was sucessfully applied to several types of robots, such as the Gough-Stewart (GS) platform, the Adept Quattro and other robots of the same family [2,3].

However, two unexpected results arose from the use of this technique: (1) it was possible to control the robot by observing a number of legs fewer than the total number of legs; this is surprising because in actuator-based control schemes, each actuated leg has to be controlled to fully servo the robot, and (2) in some cases, the robot did not converge to the desired pose, even if all observed leg directions did.

Not only were these two points inexplicable, but other questions arose too: (3) are we sure there are no local minima (for which the error in the observation space is non zero while the robot platform cannot move [8]) and (4) are we sure that there is no singularity in the mapping between the leg direction and the Cartesian spaces?

Due to the unusual nature of this visual servoing technique, all these points were left unanswered. Indeed, the nature of the mapping was not clearly understood. The answer came only recently, when two of the authors of the present paper proposed the existence of a virtual robot model "hidden" within the controller. This robot presents singular configurations and assembly modes different from the controlled robot, and it is this hidden robot whose properties are being used through the observation of the real robot's leg directions. This proposition was fully demonstrated and validated through experiments in [13].

The aim of the present paper is to introduce the generalized concept of hidden robot model and to show why the tools used in mechanical design can be efficently applied in control analysis and how the hidden robot concept must modify the robot design methodologies in order to include control-based performance indices.

\section{Leg observation based control}

\subsection{Cylindrical leg observation and Interaction matrix}

The controller proposed in [2] is based on the fact that the robot leg directions ${ }^{c} \underline{\mathbf{u}}_{i}$ (extracted from the projection of the cylindrical link in the image space - Fig. 1) are controlled and that the leg direction velocities can be linked to the platform twist ${ }^{c} \tau_{c}$ through the expression (the superscript " $c$ " denotes the camera frame):

$$
{ }^{c} \dot{\mathbf{u}}_{i}=\mathbf{M}_{i}^{T c} \tau_{c}
$$

where $\mathbf{M}_{i}^{T}$ is the $(3 \times 6)$ interaction matrix for the leg $i$. 


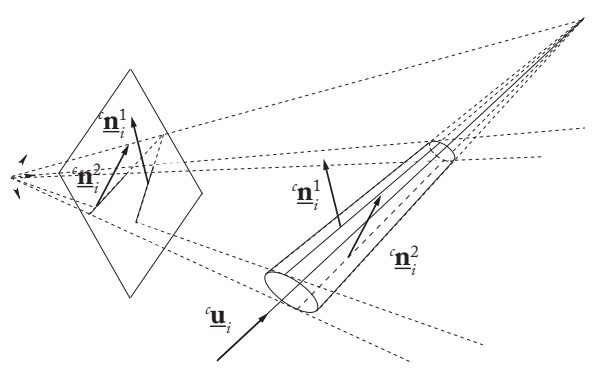

Fig. 1 Projection of a cylinder in the image

For spatial parallel robots, matrices $\mathbf{M}_{i}$ are in general of rank 2 [2]. As a result, for spatial robots with more than 2 dof, the observation of several independent legs is necessary to control the end-effector pose. An interaction matrix $\mathbf{M}^{T}$ can be obtained by stacking $k$ matrices $\mathbf{M}_{i}^{T}$ of $k$ legs, which leads to:

$$
{ }^{c} \underline{\dot{\mathbf{u}}}=\left[\underline{\underline{\mathbf{u}}}_{i}{ }^{c} \underline{\dot{\mathbf{u}}}_{j}, \ldots,{ }^{c} \underline{\mathbf{u}}_{m}\right]^{T}=\left[\mathbf{M}_{i}, \mathbf{M}_{j}, \ldots, \mathbf{M}_{m}\right]^{T}{ }^{c} \tau_{c}=\mathbf{M}^{T c} \tau_{c}
$$

\subsection{Control}

For the visual servoing of a robot, one achieves exponential decay of an error $e\left(s, s_{d}\right)$ between the current primitive vector $s$ and the desired one $s_{d}$ using a proportional linearizing and decoupling control scheme. The visual primitives being unit vectors, it is more elegant to use the geodesic error rather than the standard vector difference, i.e. the error grounding the proposed control law will be $\mathbf{e}_{i}={ }^{c} \underline{\mathbf{u}}_{i} \times{ }^{c} \underline{\mathbf{u}}_{d i}$, where ${ }^{c} \underline{\mathbf{u}}_{d i}$ is the desired value of ${ }^{c} \underline{\mathbf{u}}_{i}$. A control is then chosen such that $\mathbf{e}$, the vector stacking the errors $\mathbf{e}_{i}$ associated to of $k$ legs, decreases exponentially (i.e. $\dot{\mathbf{e}}=-\lambda \mathbf{e}$ ).

Then, introducing $\mathbf{L}_{i}^{T}=-\left[{ }^{c} \underline{\mathbf{u}}_{d i}\right]_{\times} \mathbf{M}_{i}^{T}$, where $\left[{ }^{c} \underline{\mathbf{u}}_{d i}\right]_{\times}$is the cross product matrix associated with the vector ${ }^{c} \underline{\mathbf{u}}_{d i}$, the control law can be derived:

$$
{ }^{c} \tau_{c}=-\lambda \mathbf{L}^{T+} \mathbf{e}
$$

where $\mathbf{L}^{T}$ can be obtained by stacking the matrices $\mathbf{L}_{i}^{T}$ of $k$ legs and the upperscript "+" corresponds to the matrix pseudo-inverse.

This expression can be transformed into the control joint velocities:

$$
\dot{\mathbf{q}}=-\lambda^{c} \mathbf{J}^{i n v} \mathbf{L}^{T+} \mathbf{e}
$$

where ${ }^{c} \mathbf{J}^{i n v}$ is the inverse kinematic matrix of the robot relating the end-effector twist to the actuator velocities, i.e. ${ }^{c} \mathbf{J}^{i n v c} \tau_{c}=\dot{\mathbf{q}}$.

It can be proven that the controller can meet numerical issues if: 


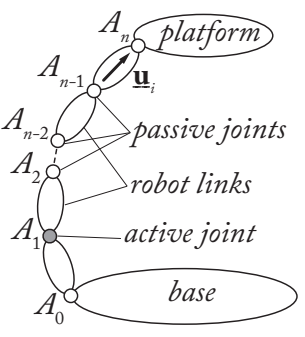

(a) A general robot leg

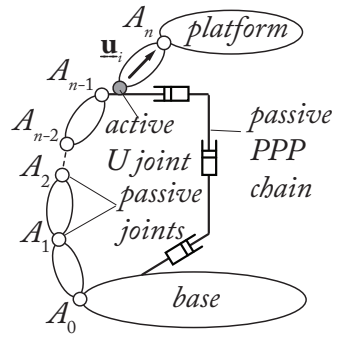

(b) its corresponding hidden robot leg when the vector $\underline{\mathbf{u}}_{i}$ is observed

Fig. 2 A general robot leg and its corresponding hidden robot leg when the vector $\underline{\mathbf{u}}_{i}$ is observed

- the matrix $\mathbf{L}^{T}$ is rank deficient: in that case, a null error vector $\mathbf{e}$ can lead to a non null platform twist ${ }^{c} \tau_{c}$. This appears if and only if $\mathbf{M}^{T}$ is rank deficient.

- the pseudo-inverse $\mathbf{L}^{T+}$ is rank deficient: in that case, we can meet a local minimum of the controller [8] for which the value of the platform twist ${ }^{c} \tau_{c}$ is zero while the error $\mathbf{e}$ is not. This appears if and only if $\mathbf{M}^{T+}$ is rank deficient.

Clearly, only stacking leg interaction matrices (which is usual in the visual servoing community) is not enough and the singularities of the controller must be studied. The rank-deficiency conditions are difficult to analyze, and indeed, the complexity for the formerly studied robots was so high that they were never obtained. It is shown in the next part that they can be analyzed by taking into account that the matrix $\mathbf{M}^{T}$ is indeed the inverse kinematic matrix of a virtual robot hidden into the controller.

\section{The concept of hidden robot model}

\subsection{How to define the legs of the hidden robots}

The concept of hidden robot model comes from the following observation: in the classical control approach, the encoders measure the motion of the actuators and this measure is linked to the forward kinematic problem $(f k p) \mathbf{x}=\mathscr{H}(\mathbf{q})$, where $\mathbf{x}$ represents the platform pose and $\mathbf{q}$ the encoder measures; in the previously described control approach, the leg directions are observed. So, in a reciprocal manner, one could wonder to what kind of virtual actuators such observations correspond, i.e. what is the virtual robot hidden below the new $f k p \mathbf{x}=\mathscr{G}(\underline{\mathbf{u}})$.

The virtual robot hidden into the controller corresponding to $\mathbf{x}=\mathscr{G}(\underline{\mathbf{u}})$ can be described as follows [13]. First, the observed links mounted to the platform must be disassembled from the rest of the robot and mounted onto active $\underline{U}$ joints (Fig. 2). The $\underline{U}$ joint must be linked to a passive kinematic chain composed of at most 3 orthogonal passive $P$ joints that ensures that the link on which is it attached performs 
a translation w.r.t. the base frame. This passive chain is also linked to the segments before the observed links so that they do not change their motion. Note that:

- it is necessary to fix the PPP chain on the preceeding leg links because the information given by the vectors $\mathbf{u}_{i}$ is not enough for rebuilding the full platform position and orientation: it is also necessary to get information on the location of the anchor point $A_{n-1}$ of the observed segment [1];

- $3 P$ joints are only necessary if and only if the point $A_{n-1}$ describes a motion in the 3D space; if not, the number of $P$ joints can be decreased such as in [6];

- when the vector $\underline{\mathbf{u}}_{i}$ is constrained to move in a plane (e.g. for planar legs), the virtual actuator becomes an $\underline{R}$ joint which must be mounted on the PPP chain.

It should be noticed that, in several cases for robots with a lower mobility, the last joint should be changed so that, if the number of observed legs is inferior to the number of real legs, the hidden robot keeps the same mobility (see [12]). Moreover, we have presented above the most general methodology, but not the most elegant. In many cases, a hidden robot leg architecture can be obtained such that less modifications w.r.t the real leg are achieved. For example, for the Quattro [12] made of $\underline{R}-\{2-$ $U U\}$ legs for which the parallelogram links are observed $(\{2-U U\}$ subchain links), the $R-P P P$ chain of the hidden robot leg (which is indeed a $\{R-P P P\}-\{2-\underline{U} U\}$ leg) could be fully-equivalently replaced by a planar parallelogram ( $\Pi$ joint $)[12]$.

\subsection{How analyzing the controllability of the servoed robots}

The aim of this Section is to show how to use the hidden robots for answering points 1 to 4 enumerated in the introduction of the paper.

Point 1: Let us consider a general parallel robot composed of 6 legs (one actuator per leg) and having six dof. Using the approach proposed in Section 3.1, each observed leg will lead to a modified hidden robot virtual leg with at least one actuated $\underline{U}$ joint that has two degrees of actuation. For controlling 6 dof, only 6 degrees of actuations are necessary, i.e. three actuated $\underline{U}$ are enough. Thus, in a general case, only three legs have to be observed to fully control the platform dof (e.g. see [6]).

Point 2: In many cases, the hidden robot may have assembly modes and singular configurations different from those of the real robot. If the initial and final robot configurations are not included in the same aspect [11], the robot will not be able to converge to the desired pose, but to a pose that corresponds to another assembly mode that has the same leg directions as the desired final pose (e.g. see [6, 12]).

Point 3: The interaction matrix $\mathbf{M}^{T}$ involved in the controller is the inverse kinematic matrix of the hidden robot (and, consequently, $\mathbf{M}^{T+}$ is the hidden robot kinematic matrix) which, is most of cases, is not free of singularities. Thus, finding the condition for the rank-deficiency of $\mathbf{M}^{T}$ and $\mathbf{M}^{T+}$ is equivalent to find the Type 1 and 2 (also called serial and parallel) singularities of the hidden robot [10].

Point 4: The robot could converge to local minima if the matrix $\mathbf{M}^{T+}$ is rank deficient, i.e. the hidden robot model encounters a Type 1 singularity. 


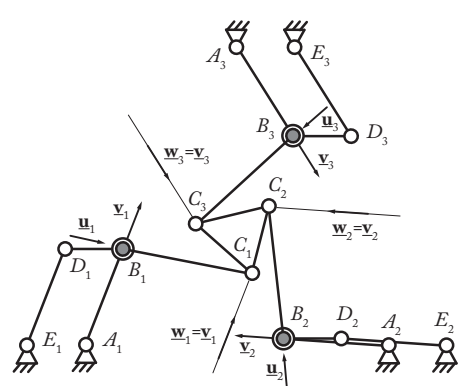

(a) $3-\Pi \underline{R R}$ robot kinematics

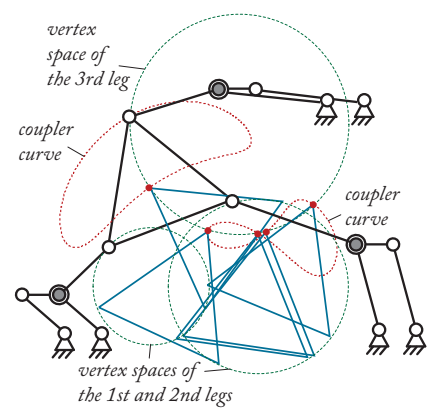

(b) The 6 possible assembly modes

Fig. 3 The hidden robot model of the $3-\underline{R R} R$ robot and its hidden robot model

Case Study: In the previous papers, only spatial mechanisms have been studied. For illustrating that section, we have decided to consider the case of the well-known planar 3-RRR robot controlled via the observation of the distal links (links between the passive $R$ joints on each leg). Using the results of previous section, it can be found that its equivalent hidden robot model is a $3-\Pi \underline{R} R$ robot (Fig. 3(a) - grey joints denote actuated joints). Each of its legs is composed of a passive planar parallelogram ( $\Pi$ joint $)$ which is able to maintain constant the orientation of the links $B_{i} D_{i}$ w.r.t. the base and of an $\underline{R} R$ chain which is mounted on the link $B_{i} D_{i}$.

Forward kinematics and assembly modes. All the solutions to the $f k p$ are at the intersections of the coupler curve (which represents the displacement loci of one platform extremity when one of the leg is disassembled, the actuators of the two other being fixed (see Fig. 3(b)) - for the present robot, the coupler curve is a sextic curve) with the vertex space of the disassembled leg (here, a circle) [11]. Thus, the solutions of the $f k p$ are at the intersection points (which are at most 6 [11]) between the aforementioned circle and sextic curve (Fig. 3(b)).

Singular configurations. The Type 1 singularities of $3-\Pi \underline{R} R$ robot appear when one leg is fully streched or folded. Its Type 2 singularities appear when the lines of direction $\underline{\mathbf{w}}_{i}(i=1,2,3-$ Fig. $3(\mathrm{a}))$ passing through points $C_{i}$ intersects in the same point (that can be at infinity). Those conditions are different from the Type 2 singular configurations of the $3-\Pi \underline{R} R$ robot, for which they appear when all lines passing through $C_{i}$ of direction $\underline{\mathbf{u}}_{i}$ intersects in one point [5].

\section{Rethinking the robot design by using the hidden robot concept}

Through the concept of hidden robot, the tools and methodology used in mechanical design - for solving the $f k p$ (e.g. [7,11], etc.), for analyzing the singularities (e.g. [4, 11], etc.) - can be efficiently used in control design and analysis. However, this concept can also be used to rethink the design process of robots controlled via the use of exteroceptive sensors: the concept of hidden robot can lead to the definition of control-based performance indices. We want to mention that, even if we have 


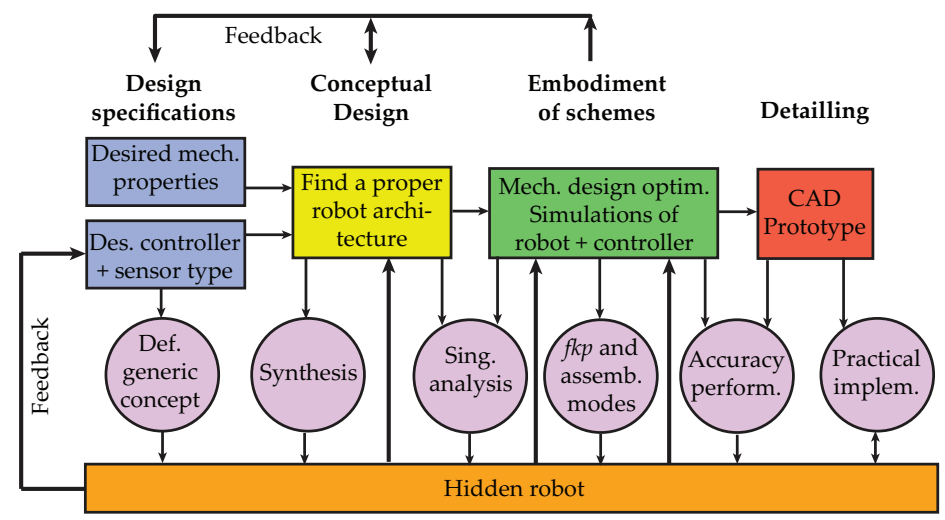

Fig. 4 How the hidden robot concept should be taken into account into the robot design process

deliberately limit the approach to the leg-based visual servoing of parallel robots, the hidden robot concept can be extended to any types of robots controlled via the use of exteroceptive sensors (e.g. cameras, lasers, etc.) which are observing robot internal motions in order to estimate the robot external properties (end-effector pose).

Figure 4 illustrates that point. The design process is typically separated into four main phases: (1) the specification of the product requirements coming from the need definition, (2) the phase of conceptual design during which concepts are proposed and evaluated, (3) the embodiement of schemes during which the concepts are developed and analyzed, and (4) the detailed design that leads to the CAD drawing and the manufacturing of prototypes. Phases (1) to (3) are obviously linked in order to modify / improve the design solutions in case of feasability issues.

If, for a given application, it is specified that a robot must be controlled via the use of exteroceptive sensors observing its legs, the designer has to know that such a requirement leads to the definition, for any kind of robot architecture, to a generic concept of hidden robot (such as the one in Fig. 2(b) for leg-based visual servoing). Then, during the conceptual design phase, for each proposed robot architecture, the corresponding hidden robots can be defined and their singularities can be analyzed. A feedback can be given to the designer so that he can choose, for example, the robot architectures that have the hidden robot models with the minimal number of singular configurations. If, for any reason, all hidden robots are architecturally singular, the design specifications concerning sensor types and observed elements must be modified (by proposing other sensors, or other observed elements). Then, once the potential architectures are selected, they must be optimized in phase 3. More detailed kinematic models of the robots and of their corresponding hidden robots are defined and used for extracting performances indices (e.g. w.r.t. accuracy), for defining a controller, and simulating the robot behavior. If the results do not meet the design requirements, previous design steps must be conducted again. Finally, during the implementation of the controller in the manufactured prototype, the hid- 
den robot model equations must be implemented. Our future works will focus on the practical implementation of such a design methodology.

\section{Conclusion}

In that paper, it has been shown that some sensor-based control approaches well adapted for servoing parallel robots involve the presence of virtual robots hidden into the controller, which have assembly modes and singularities different from the real robots. The analysis of the robot models hidden into the controllers developped by the sensor-based control community required the use of mathematical tools developped by the mechanical design community. This analysis is crucial to avoid control issues that can arise if interaction matrices are stacked without deeper analysis. It has also been shown that the tools used in mechanical design can be efficently used in control analysis and that the hidden robot concept can be smartly used in the design process of robots in order to include control-based performance indices.

\section{References}

1. Andreff, N., Dallej, T., Martinet, P.: Image-based visual servoing of Gough-Stewart parallel manipulators using legs observation. Int. Journal of Robotics Research 26(7), 677-687 (2007)

2. Andreff, N., Marchadier, A., Martinet, P.: Vision-based control of a Gough-Stewart parallel mechanism using legs observation. In: Proceedings of the IEEE International Conference on Robotics and Automation, ICRA'05, pp. 2546-2551. Barcelona, Spain (2005)

3. Andreff, N., Martinet, P.: Vision-based kinematic modelling of some parallel manipulators for control purposes. In: Proceedings of EuCoMeS. Obergurgl, Austria (2006)

4. Ben-Horin, P., Shoham, M.: Singularity analysis of a class of parallel robots based on grassmanncayley algebra. Mechanism and Machine Theory 41(8), 958-970 (2006)

5. Bonev, I., Zlatanov, D., Gosselin, C.: Singularity analysis of 3-dof planar parallel mechanisms via screw theory. ASME Journal of Mechanical Design 125(3), 573-581 (2003)

6. Briot, S., Martinet, P.: Minimal representation for the control of Gough-Stewart platforms via leg observation considering a hidden robot model. In: Proceedings of the 2013 IEEE International Conference on Robotics and Automation (ICRA 2013). Karlsruhe, Germany (2013)

7. Caro, S., Moroz, G., Gayral, T., Chablat, D., Chen, C.: Singularity analysis of a six-dof parallel manipulator using Grassmann-Cayley algebra and Groebner bases. In: Proceedings of the Symposium on Brain, Body and Machine. Montreal, QC, Canada (2010)

8. Chaumette, F.: The Confluence of Vision and Control, chap. Potential problems of stability and convergence in image-based and position-based visual servoing, pp. 66-78. No. 237 in LNCIS. Springer-Verlag (1998)

9. Espiau, B., Chaumette, F., Rives, P.: A new approach to visual servoing in robotics. IEEE Transactions on Robotics and Automation 8(3) (1992)

10. Gosselin, C., Angeles, J.: Singularity analysis of closed-loop kinematic chains. IEEE Transactions on Robotics and Automation 6(3), 281-290 (1990)

11. Merlet, J.: Parallel Robots, 2nd edn. Springer (2006)

12. Rosenzveig, V., Briot, S., Martinet, P.: Minimal representation for the control of the Adept Quattro with rigid platform via leg observation considering a hidden robot model. In: Proc. IEEE/RSJ Int. Conf. on Intelligent Rob. Syst. (IROS 2013). Tokyo Big Sight, Japan (2013)

13. Rosenzveig, V., Briot, S., Martinet, P., Ozgur, E., Bouton, N.: A method for simplifying the analysis of leg-based visual servoing of parallel robots. In: Proc. 2014 IEEE Int. Conf. on Robotics and Automation (ICRA 2014). Hong Kong, China (2014) 